\title{
STRONGLY SINGULAR INTEGRALS ON STRATIFIED GROUPS
}

\author{
PAOLO CIATTI AND JAMES WRIGHT
}

In honour of Fulvio Ricci on his 70th birthday

\begin{abstract}
We consider a class of spectral multipliers on stratified Lie groups which generalise the class of Hörmander multipliers and include multipliers with an oscillatory factor. Oscillating multipliers have been examined extensively in the euclidean setting where sharp, endpoint $L^{p}$ estimates are well known. In the Lie group setting, corresponding $L^{p}$ bounds for oscillating spectral multipliers have been established by several authors but only in the open range of exponents. In this paper we establish the endpoint $L^{p}(G)$ bound when $G$ is a stratified Lie group. More importantly we begin to address whether these estimates are sharp.
\end{abstract}

\section{INTRODUCTION}

The following class of strongly singular convolution operators on $\mathbb{R}^{n}$ given by

$$
T_{a, b} f(x)=\int_{|y| \leq 1} f(x-y) \frac{e^{i|y|^{-a}}}{|y|^{b}} d y
$$

where $a>0$ and $b \leq n(2+a) / 2$ has a rich and interesting history. In the periodic setting, they were investigated by Hardy who used them to construct a variety of counterexamples. Regarding $L^{p}$ boundedness properties, Hirschman 14 considered the one dimensional case and for general $n \geq 1$, Wainger [31 established the sharp $L^{p}$ range but left open the endpoint case which C. Fefferman and Stein [9] accomplished using interpolation by proving that $T_{a, n}$ is bounded on the Hardy space $H^{1}\left(\mathbb{R}^{n}\right)$. Earlier C. Fefferman 8 ] established that $T_{a, n}$ satisfies a weak-type $(1,1)$ bound. Chanillo [2] extended these results to weighted $L^{p}$ estimates. It is well known that when $b>n(2+a) / 2$, there are no $L^{p}$ estimates.

As a convolution operator, we can view $T=T_{a, b}$ as a multiplier operator $\widehat{T f}(\xi)=$ $m(\xi) \widehat{f}(\xi)$ where $m=m_{\theta, \beta}$ is essentially given by

$$
m_{\theta, \beta}(\xi)=\frac{e^{i|\xi|^{\theta}}}{|\xi|^{\theta / 2}}
$$

for $|\xi|$ large. Here $0<\theta=a /(1+a)<1$ and $\beta=((2+a) n-2 b) / a$. We note that $m$ is bounded precisely when $b \leq n(2+a) / 2$.

1991 Mathematics Subject Classification. 42B15; 42B20; 43A22 (primary); ; 35P99 (secondary) . 
The case $b=n$, or equivalently $\beta=n$ in (11), corresponds to the singular integral operators $T_{a, n}$, treated by Fefferman and Stein, whose convolution kernels just fail to be integrable. Their multipliers $m_{\theta, n}$ are not Hörmander multipliers but furnish examples of multipliers with $S_{\rho, \delta}^{-m}$ symbols where $m \geq 0$ and $\rho<1$. In this context these multipliers were studied by Hörmander [15].

Note that the multipliers $m_{\theta, \beta}$ in (10) with $\beta>n$ (so that $b<n$ ) correspond to operators $T_{a, b}$ with integrable convolution kernels and hence are bounded on $L^{1}$. For any $\delta>0$, consider the analytic family $T_{z}^{\delta}, \operatorname{Re}(z) \in[0,1]$, of operators with multipliers

$$
m_{z}^{\delta}(\xi)=\frac{e^{i|\xi|^{\theta}}}{|\xi|^{[\theta(n+\delta) / 2] z}} \chi(\xi)
$$

where $\chi(\xi)=0$ when $|\xi| \leq 1$. Thus $T_{z}^{\delta}$ is bounded on $L^{2}$ when $z=i y$ with $\left\|T_{i y}^{\delta}\right\|_{2 \rightarrow 2}$ uniformly bounded in $y \in \mathbb{R}$. Also $T_{z}^{\delta}$ is bounded on $L^{1}$ when $z=1+i y$, again with $\left\|T_{1+i y}^{\delta}\right\|_{1 \rightarrow 1}$ uniformly bounded in $y \in \mathbb{R}$. By analytic interpolation, we see that $m_{\theta, \beta}$ is an $L^{p}$ multiplier in the open range $|1 / p-1 / 2|<\beta / 2 n$. To establish endpoint bounds, one needs to say something about the endpoint multipliers $m_{\theta, n}$ (the case $z=1$ and $\delta=0$ in (2) ). More precisely in 9, Fefferman and Stein show that multipliers $m_{1+i t}^{0}$ in (2) are $H^{1}$ multipliers with an operator norm at most $(1+|y|)^{n+1}$.

Fefferman and Stein developed a more general theory of multipliers which include the examples (1) as special cases. Let $K$ be a distribution of compact support, which is integrable away from the origin. Its Fourier transform $\widehat{K}$ is of course a function. We make the following assumptions:

$$
\left\{\begin{array}{l}
\int_{|x|>2|y|^{1-\theta}}|K(x-y)-K(x)| d x \leq B, \quad 0<|y| \leq 1, \\
|\widehat{K}(\xi)| \leq B(1+|\xi|)^{-\theta n / 2} .
\end{array}\right.
$$

In [9], Fefferman and Stein show if $K$ satisfies (3), then $|\xi|^{(n-\beta) \theta / 2} \widehat{K}(\xi), 0 \leq \beta<n$, is an $L^{p}\left(\mathbb{R}^{n}\right)$ multiplier when $|1 / p-1 / 2| \leq \beta / 2 n$. See [30] where this result is established in the open range $|1 / p-1 / 2|<\beta / 2 n$.

In the papers 3] and [4] (see also 21]), Chanillo, Kurtz and Sampson considered the cases $\theta>1$ and $\theta<0$ (here the $|\xi|$ large restriction becomes $|\xi|$ small). Hence multipliers on $\mathbb{R}^{n}$ of the form

$$
m_{\theta, \beta}(\xi)=\frac{e^{i|\xi|^{\theta}}}{|\xi|^{\theta \beta / 2}} \chi_{ \pm}(\xi)
$$

for any $\theta \in \mathbb{R}$ and $\beta \geq 0$ have been studied. Here $\chi_{+}(\xi) \equiv 0$ for $|\xi| \leq 1$ when $\theta>0$ and $\chi_{-}(\xi) \equiv 0$ when $|\xi| \geq 1$ when $\theta<0$.

The case $\theta=1$ is special and is related to the wave operator. The sharp range of $L^{p}$ bounds in this case is different from the case $\theta \neq 1$; see [26] and 22]. We will not consider the case $\theta=1$ and assume always $\theta \neq 1$.

In this paper we will put all these oscillating multipliers into a single, general framework (much like what Fefferman and Stein do in (3) when $0<\theta<1$ ) which 
strictly generalises the class of Hörmander multipliers and furthermore we will give a unified, purely spectral treatment which readily extends to estimates for corresponding spectral multipliers on any stratified Lie group.

1.1. Notation. Keeping track of constants and how they depend on the various parameters will be important for us. For the most part, constants $C$ appearing in inequalities $P \leq C Q$ between positive quantities $P$ and $Q$ will be absolute or uniform in that they can be taken to be independent of the parameters of the underlying problem. We will use $P \lesssim Q$ to denote $P \leq C Q$ and $P \sim Q$ to denote $C^{-1} Q \leq P \leq C Q$. Furthermore, we use $P \ll Q$ to denote $P \leq \delta Q$ for a sufficiently small constant $\delta>0$ whose smallness will depend on the context.

Acknowledgement: We woud like to thank Alessio Martini and Steve Wainger for discussing the history of the problem as well as guiding us through the literature.

\section{The EUCLIDEAN SETting $\mathbb{R}^{n}$}

We start in the euclidean setting $\mathbb{R}^{n}$. Let $\phi \in C_{0}^{\infty}\left(\mathbb{R}^{n}\right)$ be supported away from the origin and let $m^{j}(\xi):=m\left(2^{j} \xi\right) \phi(\xi)$. It is natural to impose conditions on the $j$ th pieces $m^{j}$. The classical Hörmander condition requires uniform (in $j$ ) control of some $L^{2}$ Sobolev norm $\left\|m^{j}\right\|_{L_{s}^{2}}$ with $s$ derivatives. Here we want to consider not only classical Hörmander multipliers but also oscillating multipliers $m_{\theta, \beta}$ described in (4). Special among these are the endpoint multipliers $m_{\theta, n}$ whose bounds we interpolate with trivial $L^{2}$ bounds to deduce sharp $L^{p}$ bounds for $m_{\theta, \beta}$ for general $\beta \geq 0$. Hence our conditions will not only involve a smoothness parameter $s>0$ but also an oscillation parameter $\theta \in \mathbb{R}$ and a decay parameter $\beta \geq 0$.

For any $\theta \in \mathbb{R}$, the condition $j \theta>0$ identifies the frequency range of interest. In fact if $\theta>0$, then $j \theta>0$ corresponds to $j>0$ or $|\xi| \geq 1$ which is the relevant frequency range indicated in (4). However if $\theta<0$, then $j \theta>0$ corresponds to $j<0$ or $|\xi| \leq 1$ which is the range of interest for the oscillating multipliers in (4) with $\theta<0$. Finally when $\theta=0$, the condition $j \theta>0$ is vacuous.

2.1. Our multiplier conditions. We consider the following conditions on a multiplier $m$ which will depend on parameters $s, \theta$ and $\beta$. When $j \theta \leq 0$, we impose the standard uniform $L^{2}$ Sobolev norm control on the $m_{j}$;

$$
\sup _{j: j \theta \leq 0}\left\|m^{j}\right\|_{L_{s}^{2}\left(\mathbb{R}^{n}\right)}<\infty .
$$

For $j \theta>0$, we consider the condition

$$
\sup _{j: j \theta>0} 2^{j \theta \beta / 2}\left\|m^{j}\right\|_{L^{\infty}\left(\mathbb{R}^{n}\right)}, \quad 2^{-j \theta(2 s-\beta) / 2}\left\|m^{j}\right\|_{L_{s}^{2}\left(\mathbb{R}^{n}\right)}<\infty .
$$

When $\theta=0$, the condition (6) is vacuous and (5) reduces to the condition $\sup _{j}\left\|m^{j}\right\|_{L_{s}^{2}}<$ $\infty$ and if this holds for some $s>n / 2$, the classical Hörmander theorem states that the multiplier operator is of weak-type $(1,1)$ and maps $H^{1}\left(\mathbb{R}^{n}\right)$ boundedly into $L^{1}\left(\mathbb{R}^{n}\right)$. See [29. 
One can easily verify that the conditions (5) and (6) are satisfied for $m_{\theta, \beta}$ in (4) and for all $s>0$. Note that in (6), the quantity $j \theta$ is always positive and so (6) expresses a growth in the Sobolev norm $L_{s}^{2}$ of $m^{j}$ (when $s>\beta / 2$ ) and a decay in the $L^{2}$ norm of $m^{j}$. If the condition (6) is satisfied for some $s>0$, it does not necessarily hold for all $s^{\prime} \leq s$. Therefore we introduce $M_{\theta, \beta, s}$ consisting of those functions $m$ which satisfiy (5) with exponent $s$ and satisfies (6) for all exponents $s^{\prime} \leq s$.

2.2. Our multiplier classes. Hence $\cup_{s>n / 2} M_{0, *, s}$ is the classical class of Hörmander multipliers and so

$$
\mathcal{M}_{n}:=\bigcup_{\theta \in \mathbb{R} \backslash\{1\}, s>n / 2} M_{\theta, n, s}
$$

gives us a natural extension of Hörmander multipliers. It is easy to verify that the conditions (5) and (6) are independent on the choice of bump function $\phi$ and hence for any $\beta \geq 0$,

$$
\mathcal{M}_{\beta}:=\bigcup_{\theta \in \mathbb{R} \backslash\{1\}, s>n / 2} M_{\theta, \beta, s}=\left\{|\xi|^{(n-\beta) \theta / 2} m(\xi): m \in \mathcal{M}_{n}\right\} .
$$

This puts us in the position to employ the analytic interpolation argument in 9 ] to deduce that $m \in \mathcal{M}_{\beta}$ is an $L^{p}$ multiplier in the sharp range $|1 / p-1 / 2| \leq \beta / 2 n$ from $H^{1}$ bounds for multiplier operators associated to $m \in \mathcal{M}_{n}$.

In fact one advantage of working with $\mathcal{M}_{n}$ (over say, the class of multipliers arising from kernels satisfying (3) in the case $0<\theta<1$ ) is the class $\mathcal{M}_{n}$ has the desirable property that it is invariant under multiplication by $|\xi|^{i y}$ for any real $y \in \mathbb{R}$; that is, if $m \in \mathcal{M}_{n}$, then $|\xi|^{i y} m(\xi)$ lies in $\mathcal{M}_{n}$, satisfying the bounds (5) and (6) with polynomial growth in $|y|$. Hence for the analytic interpolation argument, we only need to establish that multipliers in $\mathcal{M}_{n}$ map $H^{1}$ to $L^{1}$ instead of showing they map $H^{1}$ to $H^{1}$ as needed in [9]. This will be particularly useful when we move to the setting of Lie groups.

2.3. The basic decomposition. When we analyse a multiplier $m \in \mathcal{M}_{\beta}$, we will decompose $m=\sum_{j} m_{j}$ where $m_{j}(\xi)=m(\xi) \phi\left(2^{-j} \xi\right)$ for some $\phi \in C_{0}^{\infty}\left(\mathbb{R}^{n}\right)$ supported away from the origin such that $\sum_{j} \phi\left(2^{-j} \xi\right)=1$ for all $\xi \neq 0$. Note that $m^{j}(\xi)=m_{j}\left(2^{j} \xi\right)$ is the $j$ th piece on which we impose the conditions (5) and (6). We split the multiplier $m=m_{\text {small }}+m_{\text {large }}$ into two parts where

$$
m_{\text {small }}(\xi):=\sum_{j: j \theta \leq 0} m_{j}(\xi) \text { and } m_{\text {large }}(\xi):=\sum_{j: j \theta>0} m_{j}(\xi) .
$$

If $\theta=0$, then $m=m_{\text {small }}$ and in general we note that $m_{\text {small }}$ is a Hörmander multipler (since (5) holds for some $s>n / 2$ ) and so it is an $L^{p}$ multiplier for all $1<p<\infty$ (as well as a weak-type $(1,1)$ and an $H^{1}$ multiplier). We introduce the notation $\mathcal{K}_{F}$ to denote the convolution kernel associated to a multiplier $F$. Hence it suffices to treat the operator

$$
T^{l} f(x)=\sum_{j: j \theta>0} \mathcal{K}_{m_{j}} * f(x)=: \mathcal{K}^{l} * f(x)
$$


corresponding to the interesting frequency range where the $j$ th pieces $m^{j}$ satisfy (6).

2.4. $\mathcal{M}_{n}$ versus (3). When $m \in M_{\theta, n, s} \subset \mathcal{M}_{n}$ for $0<\theta<1$, we claim that $\mathcal{K}^{l}$ satisfies the condition (3) of Fefferman and Stein in [9] (see also 30]). Hence for $0<\theta<1$, the class of convolution operators satisfying (3) is larger than the class $\mathcal{M}_{n}$. In fact the $L^{\infty}$ condition on the $m^{j}$ in (6) is equivalent to the bound $\left|\widehat{\mathcal{K}}^{l}(\xi)\right| \leq B(1+|\xi|)^{-\beta n / 2}$. Furthermore we bound

$$
\int_{|x| \geq 2|y|^{1-\theta}}\left|\mathcal{K}^{l}(x-y)-\mathcal{K}^{l}(x)\right| d x \leq \sum_{j>0} \int_{|x| \geq 2|y|^{1-\theta}}\left|\mathcal{K}_{m_{j}}(x-y)-\mathcal{K}_{m_{j}}(x)\right| d x
$$

and split the sum on the right $\sum_{j \in J_{1}}+\sum_{j \in J_{2}}$ where $J_{1}=\left\{j>0: 2^{j} \geq|y|^{-1}\right\}$ and $J_{2}=\mathbb{N} \backslash J_{1}$. For the sum over $J_{1}$, we bound each

$$
\int_{|x| \geq 2|y|^{1-\theta}}\left|\mathcal{K}_{m_{j}}(x-y)-\mathcal{K}_{m_{j}}(x)\right| d x \leq 2 \int_{|x| \geq|y|^{1-\theta}}\left|\mathcal{K}_{m_{j}}(x)\right| d x
$$

and note that if $s>n / 2$,

$$
\begin{gathered}
\int_{|x| \geq|y|^{1-\theta}}\left|\mathcal{K}_{m_{j}}(x)\right| d x=\int_{|x| \geq 2^{j}|y|^{1-\theta}}\left|\mathcal{K}_{m^{j}}(x)\right| d x=\int_{|x| \geq 2^{j}|y|^{1-\theta}}\left|\mathcal{K}_{m^{j}}(x)\right||x|^{s}|x|^{-s} d x \\
\lesssim\left(2^{j}|y|^{1-\theta}\right)^{-(s-n / 2)}\left\|m^{j}\right\|_{L_{s}^{2}} \lesssim\left(2^{j}|y|\right)^{-(1-\theta)(s-n / 2)}
\end{gathered}
$$

by Cauchy-Schwarz and (6). This is summable for $j \in J_{1}$ leaving us to treat the sum over $J_{2}$. In this case we bound

$$
\int_{|x| \geq 2|y|^{1-\theta}}\left|\mathcal{K}_{m_{j}}(x-y)-\mathcal{K}_{m_{j}}(x)\right| d x \leq|y| \int_{|x| \geq|y|^{1-\theta}}\left|\nabla \mathcal{K}_{m_{j}}(x)\right| d x
$$

and note that

$$
\nabla \mathcal{K}_{m^{j}}(x)=\int i \xi \phi(\xi) m\left(2^{j} \xi\right) e^{i x \cdot \xi} d \xi=: \int \psi(\xi) m\left(2^{j} \xi\right) e^{i x \cdot \xi} d \xi
$$

for some $\psi \in C_{0}^{\infty}\left(\mathbb{R}^{n}\right)$ supported away from 0 . Therefore $\nabla \mathcal{K}_{m^{j}}$ satisfies the bounds in (6). We write

$$
\begin{gathered}
\int_{|x| \geq|y|^{1-\theta}}\left|\nabla \mathcal{K}_{m_{j}}(x)\right| d x=2^{j} \int_{|x| \geq 2^{j}|y|^{1-\theta}}\left|\nabla \mathcal{K}_{m^{j}}(x)\right| d x \\
=2^{j} \int_{2^{j}|y|^{1-\theta} \leq|x| \leq 2^{j \theta}}\left|\nabla \mathcal{K}_{m^{j}}(x)\right| d x+2^{j} \int_{2^{j \theta} \leq|x|}\left|\nabla \mathcal{K}_{m^{j}}(x)\right| d x=: I_{j}+I I_{j} .
\end{gathered}
$$

We note that the integration in $I_{j}$ is nonempty since $|y|^{1-\theta} \leq 2^{-j(1-\theta)}$ for $j \in J_{2}$. By Cauchy-Schwarz and (6) we have

$$
I_{j} \leq 2^{j} 2^{j \theta n / 2}\left\|\mathcal{K}_{m^{j}}\right\|_{L^{2}}=2^{j} 2^{j \theta n / 2}\left\|m^{j}\right\|_{L^{2}} \lesssim 2^{j} 2^{j \theta n / 2}\left\|m^{j}\right\|_{L^{\infty}} \lesssim 2^{j} .
$$

In precisely the same way we argued in (9) we also have $\left|I I_{j}\right| \lesssim 2^{j}$. Hence $\sum_{j \in J_{2}} \mid I_{j}+$ $\left.I I_{j}|\lesssim| y\right|^{-1}$ and this shows that we can sum the integrals in (10) and get a uniform bound, establishing the claim that (3) holds for $\mathcal{K}^{l}$. 
2.5. An interlude. At this point we would like to highlight a useful bound which is trivial in the euclidean setting but will not be so trivial in the Lie group setting. The following bound is an immediate consequence of the Cauchy-Schwarz inequality:

For any compactly support $F$ with $\operatorname{supp}(F) \subseteq K$ ( $K$ compact $)$,

$$
\left\|\mathcal{K}_{F}\right\|_{L^{1}\left(\mathbb{R}^{n}\right)} d x \leq C_{s, K}\|F\|_{L_{s}^{2}\left(\mathbb{R}^{n}\right)}
$$

holds for any $s>n / 2$.

We can use (11) to conclude that if the decay parameter $\beta>n$, then the main part of the convolution kernel $\mathcal{K}^{l}$ is integrable for any $m \in \mathcal{M}_{\beta}$. To see this, note that $m \in M_{\theta, \beta, s}$ for some $\theta \in \mathbb{R}$ and $s>n / 2$, and by (11),

$$
\left\|\mathcal{K}^{l}\right\|_{L^{1}} \leq \sum_{j: j \theta>0}\left\|\mathcal{K}_{m_{j}}\right\|_{L^{1}}=\sum_{j: j \theta>0}\left\|\mathcal{K}_{m^{j}}\right\|_{L^{1}} \lesssim \sum_{j: j \theta>0}\left\|m^{j}\right\|_{L_{s^{\prime}}^{2}} \lesssim \sum_{j: j \theta>0} 2^{-j \theta\left(\beta-2 s^{\prime}\right) / 2}
$$

for any $s^{\prime}>n / 2$. Since $\beta>n$ and $s>n / 2$, we can find an $s^{\prime} \leq s$ such that $n / 2<$ $s^{\prime}<\beta / 2$. Hence the above sum is convergent and this shows that $\mathcal{K}^{l} \in L^{1}\left(\mathbb{R}^{n}\right)$.

By embedding a general $m \in \mathcal{M}_{\beta}$ with $0 \leq \beta<n$ into the analytic family of multipliers $m_{z}(\xi)=|\xi|^{\theta / 2(\beta-(n+\delta) z)} m(\xi)$ (see (2) ) and using analytic interpolation, we have the following observation.

Lemma 2.6. If $m \in \mathcal{M}_{\beta}$ and $0 \leq \beta<n$, then $m$ is an $L^{p}\left(\mathbb{R}^{n}\right)$ multiplier if $|1 / p-1 / 2|<\beta / 2 n$.

Lemma 2.6 is an extension of a result in 30 from the case $0<\theta<1$ to the case of general $\theta \neq 1$.

2.7. The results. As discussed above, using (7) and the analytic interpolation argument in [9], we can show that any $m \in \mathcal{M}_{\beta}$ with $0 \leq \beta<n$ is an $L^{p}$ multiplier at the endpoint $|1 / p-1 / 2|=\beta / 2 n$ IF we can show that every endpoint multiplier $m \in \mathcal{M}_{n}$ is bounded from $H^{1}\left(\mathbb{R}^{n}\right)$ to $L^{1}\left(\mathbb{R}^{n}\right)$. We have the following theorem.

Theorem 2.8. For every $m \in \mathcal{M}_{n}$, the corresponding multiplier operator $T_{m}$ is weak-type $(1,1)$ and maps $H^{1}\left(\mathbb{R}^{n}\right)$ to $L^{1}\left(\mathbb{R}^{n}\right)$.

We do not claim that Theorem 2.8 is really new. For the examples in (4), Theorem 2.8 was established in the series of papers [8, [9], 3], 4] and [21] for various cases of $\theta \in \mathbb{R} \backslash\{1\}$. What is new is the proof which gives a unified approach and extends to the Lie group setting. We have the immediate consequence improving Lemma 2.6 .

Corollary 2.9. If $m \in \mathcal{M}_{\beta}$ and $0 \leq \beta<n$, then $m$ is an $L^{p}\left(\mathbb{R}^{n}\right)$ multiplier for $|1 / p-1 / 2| \leq 2 \beta / n$. 


\section{The Stratified LiE GROUP SETting}

Let $\mathfrak{g}$ be an $n$-dimensional, graded nilpotent Lie algebra so that

$$
\mathfrak{g}=\bigoplus_{i=1}^{s} \mathfrak{g}_{i}
$$

as a vector space and $\left[\mathfrak{g}_{i}, \mathfrak{g}_{j}\right] \subset \mathfrak{g}_{i+j}$ for all $i, j$. Suppose that $\mathfrak{g}_{1}$ generates $\mathfrak{g}$ as a Lie algebra. We call the associated, connected, simply connected Lie group $G$ a stratified Lie group. Associated to such a group is its so-called homogeneous dimension

$$
Q=\sum_{j} j \operatorname{dimension}\left(\mathfrak{g}_{j}\right)
$$

which is clearly always larger then the topological dimension $n$ but they agree when $G=\mathbb{R}^{n}$.

We fix a basis $\left\{X_{j}\right\}$ for $\mathfrak{g}_{1}$ where each $X_{j}$ can be identified with a unique leftinvariant vector field on $G$ which we also denote by $X_{j}$. Consider the sublaplacian $\mathcal{L}=-\sum_{k} X_{k}^{2}$ on $G$. For any Borel measurable function $m$ on $\mathbb{R}_{+}=[0, \infty)$, we can define the spectral multiplier operator

$$
m(\sqrt{\mathcal{L}})=\int_{0}^{\infty} m(\lambda) d E_{\lambda}
$$

where $\left\{E_{\lambda}\right\}_{\lambda \geq 0}$ is the spectral resolution of $\sqrt{\mathcal{L}}$. This is a bounded operator on $L^{2}(G)$ precisely when $m \in L^{\infty}\left(\mathbb{R}_{+}\right)$. The classical laplacian $\Delta$ is the corresponding differential operator when $G=\mathbb{R}^{n}$ and spectral multipliers on $\mathbb{R}^{n}$ are simply radial multipliers which the multipliers in (4) provide specific examples.

3.1. The multiplier classes. We now state the conditions corresponding to (5) and (6) for spectral multipliers $m$ defined on $\mathbb{R}_{+}$. Fix a smooth bump function $\phi$ on $\mathbb{R}$ supported away from the origin and let $m^{j}(\lambda):=m\left(2^{j} \lambda\right) \phi(\lambda)$. Again the conditions will depend on an oscillation parameter $\theta \in \mathbb{R}$, a decay parameter $\beta \geq 0$ and a smoothness parameter $s>0$. When $j \theta \leq 0$, we impose the standard uniform $L^{2}$ Sobolev norm control on the $m_{j}$;

$$
\sup _{j: j \theta \leq 0}\left\|m^{j}\right\|_{L_{s}^{2}\left(\mathbb{R}_{+}\right)}<\infty .
$$

For $j \theta>0$, we consider the condition

$$
\sup _{j: j \theta>0} 2^{j \theta \beta / 2}\left\|m^{j}\right\|_{L^{\infty}\left(\mathbb{R}_{+}\right)}, \quad 2^{-j \theta(2 s-\beta) / 2}\left\|m^{j}\right\|_{L_{s}^{2}\left(\mathbb{R}_{+}\right)}<\infty .
$$

Again when $\theta=0$, these conditions reduce to the condition $\sup _{j}\left\|m^{j}\right\|_{L_{s}^{2}}<\infty$ and if this holds for some $s>Q / 2$, the fundamental work of Christ [5] and Mauceri-Meda [20] establishes that the multiplier operator is of weak-type $(1,1)$ and bounded on $H^{1}(G)$.

The examples $m_{\theta, \beta}(\lambda)=e^{i \lambda^{\theta}} \lambda^{-\theta \beta / 2} \chi_{ \pm}(\lambda)$ from (4) satisfy conditions (12) and (13). As before we let $M_{\theta, \beta, s}$ consist of those spectral functions $m$ satisfying (12) with 
exponent $s$ and satisfying (13) for all exponents $s^{\prime} \leq s$. We redefine

$$
\mathcal{M}_{\beta}\left(=\mathcal{M}_{\beta, Q}\right):=\bigcup_{\theta \in \mathbb{R} \backslash\{1\}, s>Q / 2} M_{\theta, \beta, s}=\left\{\lambda^{(Q-\beta) \theta / 2} m(\lambda): m \in \mathcal{M}_{Q}\right\}
$$

and stress the dependence of these classes on the homogeneous dimension $Q$ which we will return to later. Again this puts us in the position to employ analytic interpolation arguments to deduce that $m \in \mathcal{M}_{\beta}$ is an $L^{p}(G)$ multiplier in the range $|1 / p-1 / 2| \leq \beta / 2 Q$ from $H^{1}(G)$ bounds for multiplier operators associated to $m \in \mathcal{M}_{Q}$. Furthermore, from the invariance of $\mathcal{M}_{Q}$ under multiplication by $\lambda^{i y}$ for any real $y$ (with resulting polynomial in y bounds in (12) and (13)), it suffices to show $m(\sqrt{\mathcal{L}}): H^{1}(G) \rightarrow L^{1}(G)$ for $m \in \mathcal{M}_{Q}$.

3.2. The main result. Our main result is the following theorem.

Theorem 3.3. For any $m \in \mathcal{M}_{Q}$, the operator $m(\sqrt{\mathcal{L}}): H^{1}(G) \rightarrow L^{1}(G)$ and is weak-type $(1,1)$.

As an immediate consequence, using analytic interpolation (see above), we have the following endpoint result of Mauceri and Meda in [20. See also the work of Alexopolous [1] on general Lie groups of polynomial volume growith.

Corollary 3.4. Every $m \in \mathcal{M}_{\beta}$ with $0 \leq \beta<Q$ is an $L^{p}(G)$ multiplier in the range $|1 / p-1 / 2| \leq \beta / 2 Q$.

3.5. The interlude - revisited. We now return to the estimate (11) and examine it in the Lie group context. Again we use the notation $\mathcal{K}_{F}$ to denote the convolution kernel of the operator $m(\sqrt{\mathcal{L}})$.

Let $G$ be any stratified Lie group and suppose the following holds for some dimensional parameter $d$ : for any spectral multiplier $F(\lambda)$, supported in a compact $K \subset \mathbb{R}_{+}$,

$$
\left\|\mathcal{K}_{F}\right\|_{L^{1}(G)} d x \leq C_{s, K}\|F\|_{L_{s}^{2}\left(\mathbb{R}_{+}\right)}
$$

holds for any $s>d / 2$.

In [5] and [20], the estimate (15) was proved for $d=Q$, the homogeneous dimension, on a general stratified Lie group $G$. In fact the estimate (15) is the key estimate in their work. It is known that if (15) holds for some parameter $d$, then standard techniques allow us to deduce that if a spectral multiplier $m$ satsifies $\sup _{j}\left\|m^{j}\right\|_{L_{s}^{2}}<\infty$ for some $s>d / 2$, then $m(\sqrt{\mathcal{L}})$ is bounded on all $L^{p}(G), 1<p<\infty$ and corresponding endpoint results on $L^{1}$ hold. See for example, [17]. Hence to determine the minimal amount of smoothness required for Hörmander-type spectral multipliers, matters can be reduced to establishing (15).

The fact that one only needs to control a little more than half the topological dimension $n$ number of derivatives, $s>n / 2$, for certain Lie groups was first observed 
by Müller and Stein 24] for the Heisenberg group. The ideas in [13] can be used to establish (15) for $d=n$ on any Lie group of Heisenberg-type (alternatively, one of the main estimates in [23] imply this immediately). Furthermore (15) for $d=n$ was established by Martini and Müller [18 for step 2 stratified Lie groups with $n \leq 7$ or whose centre has dimension at most 2. In another paper [19, Martini and Müller show that (15) holds for some $d<Q$ on any step 2 stratified Lie group.

The estimate (15) also has implications for our more general multipliers satsifying (12) and (13). Instead of $\mathcal{M}_{\beta}=\mathcal{M}_{\beta, Q}$ defined in (14), let us consider

$$
\mathcal{M}_{\beta, d}:=\bigcup_{\theta \in \mathbb{R} \backslash\{1\}, s>d / 2} M_{\theta, \beta, s}
$$

depending now on a dimensional paramter $d$ which could be smaller than $Q$. Suppose now that (15) holds for some $d \leq Q$ on $G$. We can use (15) to conclude that if $\beta>d$, then any $m \in \mathcal{M}_{\beta, d}$ can be written as $m=m_{\text {small }}+m_{\text {large }}$ (see (8)) where $m_{\text {small }}$ is a Hörmander multiplier with $s>d / 2$ (and hence bounded on all $L^{p}(G), 1<p<\infty$, weak-type $(1,1)$, etc...) and $m_{\text {large }}$ is an $L^{1}(G)$ multiplier, the convolution kernel $\mathcal{K}^{l}$ associated to $m_{\text {large }}$ being integrable. This follows exactly as in the the euclidean setting.

By embedding a general $m \in \mathcal{M}_{\beta, d}$ with $0 \leq \beta<d$ into the analytic family of spectral multipliers $m_{z}(\lambda)=\lambda^{\theta / 2(\beta-(d+\delta) z)} m(\lambda)$ and using analytic interpolation, we have the following observation.

Lemma 3.6. Suppose that (15) holds on $G$ for some $d \leq Q$. If $m \in \mathcal{M}_{\beta, d}$ and $0 \leq \beta<d$, then $m$ is an $L^{p}(G)$ multiplier for $|1 / p-1 / 2|<\beta / 2 d$.

In particular on any step 2 stratified Lie group, the result of Martini and Müller in [19, establishing that (15) holds for some $d<Q$, shows that the convolution kernel $\mathcal{K}^{l}$ corresponding to the interesting frequency range of any $m \in \mathcal{M}_{Q}=\mathcal{M}_{Q, Q}$ is integrable! In this case our main result Theorem 3.3 is a consequence of the work of Christ [5] and Mauceri-Meda [20].

Hence we should view Theorem 3.3 and Corollary 3.4 as place-holders for possible endpont results. It may be the case that (15) holds for some $d<Q$ on any stratified Lie group outwith the euclidean $G=\mathbb{R}^{n}$ case. If so, our results do not say anything new outside the euclidean setting.

In a forthcoming paper, we will establish the sharp result on any Lie group of Heisenberg-type, establishing Theorem 3.3 and Corollary 3.4 with $Q$ replaced by $n$. Our analysis heavily relies on Müller and Seeger's work [23] on the wave equation in Lie groups of Heisenberg-type.

Finally we note that Theorem 3.3 implies Theorem 2.8 in the case of radial multipliers but the proof of Theorem 3.3 below easily gives a proof of Theorem 2.8. We will therefore give the proof of Theorem 3.3 only. 


\section{Preliminaries}

For background information about Calderón-Zygmund theory and spectral multipliers on stratified groups, we refer the reader to the book of Folland and Stein [10]. If $h$ is a Borel measurable function on $\mathbb{R}_{+}$, recall that $\mathcal{K}_{h}$ denotes the convolution kernel of the operator $h(\sqrt{\mathcal{L}})$ so that

$$
h(\sqrt{\mathcal{L}}) f(x)=f * \mathcal{K}_{h}(x)=\int_{G} f\left(x \cdot y^{-1}\right) \mathcal{K}_{h}(y) d y
$$

where $d y$ denotes Haar measure on $G$. Since we are identifying the Lie group $G$ with its Lie algebra $\mathfrak{g}$ via the exponential map, the Haar measure is identified with Lebesgue measure on the Lie algebra $\mathfrak{g} \simeq \mathbb{R}^{n}$.

4.1. Some basics. The stratified group $G$ comes equipped with a group of dilations $\delta_{r}: G \rightarrow G$ which are automorphisms and we fix a homogeneous norm; that is, a function $|\cdot|: G \rightarrow \mathbb{R}_{+}$, smooth away from 0 , with $|x|=0$ if and only if $x=0$ where 0 denotes the group identity, and $\left|\delta_{r} x\right|=r|x|$ for all $r \in \mathbb{R}_{+}$and $x \in G$. Also if $s>0$, then

$$
h(s \sqrt{\mathcal{L}}) f(x)=f *\left(\mathcal{K}_{h}\right)_{s}(x) \text { where }\left(\mathcal{K}_{h}\right)_{s}(x):=s^{-Q} \mathcal{K}_{h}\left(\delta_{s^{-1}} x\right) ;
$$

see [10]. Another standard fact from [10] is the following mean value theorem for Schwartz functions $\mathcal{S}$ on $G$ : if $h \in \mathcal{S}(G)$, then for any $N \geq 1$,

$$
|h(x \cdot y)-h(x)| \leq C_{N} \frac{|y|}{(1+|x|)^{N}}
$$

holds for any $y \in G$ such that $|y| \ll|x|$. We will find this useful at times. We will also find useful the following Plancherel-type identity which can be found in [5]: for $h \in L^{\infty}\left(\mathbb{R}_{+}\right)$, there is a constant $c$ such that

$$
\left\|\mathcal{K}_{h}\right\|_{L^{2}(G)}^{2}=c \int_{0}^{\infty}|h(t)|^{2} t^{Q-1} d t
$$

holds.

4.2. A weighted $L^{2}$ bound. We will use the following weighted $L^{2}$ estimate which is valid on a general stratified Lie group $G$ : if $F$ is a compactly supported spectral multiplier, then

$$
\int_{G}\left|\mathcal{K}_{F}(x)\right|^{2}\left(1+|x|^{s}\right)^{2} d x \lesssim\|F\|_{L_{s}^{2}}
$$

holds for any $s>0$. See 28]. Note that by Cauchy-Schwarz, the bound (18) immediately shows that the key estimate (15) holds for all $s>Q / 2$ on any stratified Lie group.

For the Hardy space estimate we will use (18) but we will also use this estimate with derivatives:

$$
\int_{G}\left|X_{j} K_{F(\mathcal{L})}(x)\right|^{2}\left(1+|x|^{s}\right)^{2} d x \lesssim\|F\|_{L_{s}^{2}}
$$

holds for any $s>0,1 \leq j \leq k$ and any compactly supported $F$. Here $k=$ $\operatorname{dimension}\left(\mathfrak{g}_{1}\right)$. 
4.3. Fefferman-Stein inequality. Our argument uses the Fefferman-Stein vectorvalued Hardy-Littlewood maximal function inequality in the context of stratified groups. If

$$
M f(x)=\sup _{r>0} \frac{1}{r^{Q}} \int_{|y| \leq r}\left|f\left(x \cdot y^{-1}\right)\right| d y
$$

denotes the Hardy-Littlewood maximal function on $G$, then for $1<p, q<\infty$, we have

$$
\left\|\left(\sum_{j}\left(M f_{j}\right)^{q}\right)^{1 / q}\right\|_{L^{p}(G)} \leq C_{p, q, G}\left\|\left(\sum_{j}\left|f_{j}\right|^{q}\right)^{1 / q}\right\|_{L^{p}(G)}
$$

see for example 29] or [12. We will use this inequality for $f_{j}$ a sequence of characteristic functions of balls $B=B\left(x_{B}, r_{B}\right):=\left\{y \in G:\left|y \cdot x_{B}^{-1}\right| \leq r_{B}\right\}$. We first note that if $\chi_{B}$ denotes the characteristic function of a ball $B$, then

$$
M\left(\chi_{B}\right)(x) \sim \frac{1}{\left(1+\left|\delta_{2-L(B)}\left(x \cdot x_{B}^{-1}\right)\right|\right)^{Q}}
$$

where $L(B)$ is chosen so that $2^{L(B)}=r_{B}$. Hence $M\left(\chi_{B}\right)$ is a weak approximation of the characteristic function $\chi_{B}$ itself.

4.4. Our basic decomposition. Let us recall the basic decomposition (8) in the context of spectral multipliers $m$; we choose $\phi \in C_{0}^{\infty}\left(\mathbb{R}_{+}\right)$supported in $\{1 / 2 \leq$ $\lambda \leq 2\}$ so that $\sum_{j \in \mathbb{Z}} \phi\left(2^{-j} \lambda\right)=1$ for all $\lambda>0$. Hence $m(\lambda)=\sum_{j \in \mathbb{Z}} m_{j}(\lambda)$ where $m_{j}(t):=m(t) \phi\left(2^{-j} \lambda\right)=m^{j}\left(2^{-j} \lambda\right)$ and so

$$
\mathcal{K}_{m_{j}}(x)=\mathcal{K}_{m} *\left(2^{j Q} \mathcal{K}_{\phi}\left(\delta_{2^{j}} \cdot\right)\right)(x)=\mathcal{K}_{m} *\left(\mathcal{K}_{\phi}\right)_{2^{-j}}(x) .
$$

Therefore

$$
m(\sqrt{\mathcal{L}}) f(x)=\sum_{j \in \mathbb{Z}} m_{j}(\sqrt{\mathcal{L}}) f(x)=\sum_{j \in \mathbb{Z}} f * \mathcal{K}_{m_{j}}(x) .
$$

For $m \in \mathcal{M}_{Q}$, we split the multiplier

$$
m(\lambda)=\sum_{j \in \mathbb{Z}} m_{j}(\lambda)=\sum_{j \in \mathbb{Z}} m(\lambda) \phi\left(2^{-j} \lambda\right)=m_{\text {small }}(\lambda)+m_{\text {large }}(\lambda)
$$

into two parts where $m_{\text {small }}(\lambda)=\sum_{j: j \theta \leq 0} m_{j}(\lambda)$ and $m_{\text {large }}(\lambda)=\sum_{j: j \theta>0} m_{j}(\lambda)$. Since $m$ satisfies (12) for some $s>Q / 2$, the results of Christ [5] and MauceriMeda [20] show the multiplier $m_{\text {small }}$ is weak-type $(1,1)$ and bounded on $H^{1}(G)$ (alternatively, the argument below in the case $\theta=0$ can be used to treat $m_{\text {small }}$ ). Hence it suffices to treat the operator $T:=\sum_{j: j \theta>0} m_{j}(\sqrt{\mathcal{L}})$ and in particular it will be good to keep in mind that $j \theta>0$ is always satisfied.

\section{The Proof of Theorem 3.3 - the Weak-type $(1,1)$ Bound}

We have reduced matters to bounding $T=\sum_{j: j \theta>0} m_{j}(\sqrt{\mathcal{L}})$ and our aim here is to show that

$$
|\{x \in G:|T f(x)| \geq \alpha\}| \leq \frac{C}{\alpha}\|f\|_{L^{1}(G)}
$$


holds uniformly for all $\alpha>0$ and $f \in L^{1}(G)$. We will denote by $|\cdot|$ the Haar measure on $G$ as well as the homogeneous norm (as well as the usual absolute value on $\mathbb{R}$ or $\mathbb{C}$ ). There should be no confusion.

We employ the classical Calderón-Zygmund decomposition of $f$ at height $\alpha$ on $G$ (see [10] or [29]): there exists a sequence of essentially disjoint balls $\{B=$ $\left.B\left(x_{B}, 2^{L(B)}\right)\right\}$ such that $|\cup B| \lesssim\|f\|_{L^{1}} / \alpha$. Furthermore we can decompose $f=g+b$ where $|g(x)| \lesssim \alpha$ a.e $x \in G$ and $b=\sum_{B} b_{B}$ where $\operatorname{supp}\left(b_{B}\right) \subseteq B^{*}$,

$$
\int_{G} b_{B}=0, \quad\left\|b_{B}\right\|_{L^{1}} \lesssim \alpha|B| \text { and } \sum_{B}\left\|b_{B}\right\|_{L^{1}} \lesssim\|f\|_{L^{1}} .
$$

Here and from now on, $B^{*}$ will denote a generic dilate of $B$ which is understood to be the appropriate dilate depending on the context and we may also take it to be a sufficiently large dilate when there is a need to do so.

The contribution of the bounded function $g$ to the distribution function $\mid\{x$ : $|T f(x)| \geq \alpha\} \mid$ follows in the usual way, only the $L^{2}$ boundedness of $T$ is used here (that is, only the fact that $m$ is bounded is used). To establish (23), it suffices to consider the contribution from $T$ on the function $b=\sum_{B} b_{B}$ where $f$ is large and so we write

$$
T b(x)=\sum_{(j, B) \in N} \mathcal{K}_{m_{j}} * b_{B}(x)+\sum_{(j, B) \in P} \mathcal{K}_{m_{j}} * b_{B}(x)=: \mathcal{A}(x)+\mathcal{B}(x)
$$

where

$$
N=\{(j, B): j \theta>0, j(1-\theta)+L(B) \leq 0\}
$$

and $N$ is the complementary set of pairs $(j, B)$ with $j \theta>0$.

For the sum over the pairs $(j, B) \in N$, we use $L^{2}$ estimates, the disjoint frequency supports of the $\left\{\phi\left(2^{-j} \lambda\right)\right\}$ and the smallness of $m$ on the support of $\phi\left(2^{-j} \lambda\right)$, $m \approx 2^{-\theta j Q / 2}$. Writing $\Phi_{j}(x):=\left(\mathcal{K}_{\phi}\right)_{2^{j}}(x)$, we have

$$
\begin{gathered}
\left|\left\{x:\left|\sum_{(j, B) \in N} m_{j}(\sqrt{\mathcal{L}})\left(b_{B}\right)(x)\right| \geq \alpha\right\}\right| \leq \alpha^{-2}\left\|\sum_{(j, B) \in N} m_{j}(\sqrt{\mathcal{L}})\left(b_{B}\right)\right\|_{2}^{2} \\
\lesssim \alpha^{-2} \sum_{j: j \theta>0} \| \mathcal{K}_{m} *\left(\sum_{B \in N_{j}} \Phi_{j} * b_{B}\left\|_{2}^{2} \lesssim \alpha^{-2} \sum_{j: j \theta>0} 2^{-\theta j Q}\right\| \sum_{B \in N_{j}} \Phi_{j} * b_{B} \|_{2}^{2}\right.
\end{gathered}
$$

where $N_{j}=\{B:(j, B) \in N\}$. We write the last term on the right above as $E+F$ where

$$
E:=\alpha^{-2} \sum_{j: j \theta>0} 2^{-j Q \theta}\left\|\sum_{B \in N_{j}} \Phi_{j} * b_{B} \cdot \chi_{B^{*}}\right\|_{2}^{2} \lesssim \alpha^{-2} \sum_{(j, B) \in N} 2^{-j Q \theta}\left\|\Phi_{j} * b_{B}\right\|_{2}^{2}
$$

for some appropriately large dilate $B^{*}$ of $B$ and $F$ is defined similarly with $B^{*}$ replaced by $G \backslash B^{*}$. Since $\left\|\Phi_{j} * b_{B}\right\|_{L^{2}}^{2} \leq\left\|\Phi_{j}\right\|_{L^{2}}^{2}\left\|b_{B}\right\|_{L^{1}}^{2} \lesssim\left\|\Phi_{j}\right\|_{L^{2}(G)}^{2} \alpha^{2}|B|^{2}$ and

$$
\left\|\Phi_{j}\right\|_{L^{2}(G)}^{2}=c \int_{0}^{\infty}\left|\phi\left(2^{-j} \lambda\right)\right|^{2} t^{Q-1} d t=c_{\phi} 2^{j Q}
$$

by the Plancherel formula (17), we have $\left\|\Phi_{j} * b_{B}\right\|_{L^{2}}^{2} \lesssim 2^{j Q} \alpha^{2}|B|^{2}$. Hence

$$
E \lesssim \sum_{(j, B) \in N} 2^{j Q(1-\theta)}|B|^{2} \lesssim \sum_{B}|B| \sum_{j(1-\theta)+L(B) \leq 0} 2^{Q(j(1-\theta)+L(B))} \lesssim \sum_{B}|B| \lesssim \alpha^{-1}\|f\|_{1} .
$$


Note that it is important that $\theta \neq 1$ in the above argument. This leaves us with $F$.

Using the cancellation of $b_{B}$, we have

$$
\Phi_{j} * b_{B}(x)=\int_{G}\left[\Phi_{j}\left(x \cdot y^{-1}\right)-\Phi_{j}\left(x \cdot x_{B}^{-1}\right)\right] b_{B}(y) d y .
$$

Noting that $\Phi_{j}(x)=2^{j Q} \mathcal{K}_{\phi}\left(\delta_{2^{j}} x\right)$, we have for $y \in \operatorname{supp}\left(b_{B}\right)$ and $x \notin B^{*}$,

$$
\left|\Phi_{j}\left(x \cdot y^{-1}\right)-\Phi_{j}\left(x \cdot x_{B}^{-1}\right)\right| \lesssim 2^{j Q} \frac{2^{(1-N)(j+L(B))}}{\left(1+\left|\delta_{2-L(B)}\left(x \cdot x_{B}^{-1}\right)\right|\right)^{N}}
$$

by the mean value theorem on stratified groups (16). Therefore we see that for $x \notin B^{*}$,

$$
\left|\Phi_{j} * b_{B}(x)\right| \lesssim \alpha 2^{(Q+1-N)(j+L(B))} M\left(\chi_{B}\right)(x)^{N / Q}=\alpha 2^{\epsilon(j+L(B))} M\left(\chi_{B}\right)(x)^{q}
$$

where $\epsilon=Q+1-N$ and $q=N / Q$. By choosing $N=Q+1 / 2$, we can make $\epsilon>0$ and $q>1$. This allows us to apply the Fefferman-Stein inequality (20) which yields

$$
\begin{gathered}
F \lesssim \alpha^{-2} \sum_{j: j \theta>0} 2^{-j Q \theta} \| \sum_{B \in N_{j}} \Phi_{j} * b_{B}\left(\chi_{\left.G \backslash B^{*}\right)} \|_{2}^{2}\right. \\
\lesssim \sum_{j: j \theta>0} 2^{-j Q \theta}\left\|\sum_{B \in N_{j}}\left[M\left(2^{\epsilon(j+L(B)) / q} \chi_{B}\right)\right]^{q}\right\|_{2}^{2} \lesssim \sum_{(j, B) \in N} 2^{-j Q \theta} 2^{2 \epsilon(j+L(B))}|B| \\
=\sum_{(j, B) \in N} 2^{j(1-\theta)+L(B)} 2^{-j \theta(Q-1)}|B| \leq \sum_{(j, B) \in N} 2^{j(1-\theta)+L(B)}|B| \lesssim \sum_{B}|B| \lesssim \alpha^{-1}\|f\|_{1}
\end{gathered}
$$

since $j \theta>0$ and $Q \geq 1$.

This completes the estimate for $F$ and the contribution from the pairs $(j, B) \in N$. Hence $|\{x:|\mathcal{A}(x)| \geq \alpha\}| \lesssim \alpha^{-1}\|f\|_{1}$. Again it was important that $\theta \neq 1$ in this argument. We now turn to the contribution from the pairs $(j, B) \in P$ where we will use only $L^{1}$ estimates and the $L^{2}$ Sobolev condition in (13).

Since $\left|\cup B^{*}\right| \lesssim \alpha^{-1}\|f\|_{1}$, we see that the desired estimate $|\{x:|\mathcal{B}(x)| \geq \alpha\}| \lesssim \alpha^{-1}\|f\|_{1}$ reduces matters to estimating $\left|\left\{x \notin \cup B^{*}:|\mathcal{B}(x)| \geq \alpha\right\}\right|$ which we see is at most

$$
\begin{gathered}
\alpha^{-1} \int_{x \notin \cup B^{*}}\left|\sum_{(j, B) \in P} m_{j}(\sqrt{\mathcal{L}})\left(b_{B}\right)(x)\right| d x \leq \alpha^{-1} \sum_{(j, B) \in P} \int_{x \notin B^{*}}\left|m_{j}(\sqrt{\mathcal{L}})\left(b_{B}\right)(x)\right| d x \\
\leq \alpha^{-1} \sum_{(j, B) \in P} \int\left|b_{B}(y)\right|\left[\int_{\left|x \cdot x_{B}^{-1}\right| \gg 2^{L(B)}}\left|\mathcal{K}_{m_{j}}\left(x \cdot y^{-1}\right)\right| d x\right] d y .
\end{gathered}
$$

The desired estimate will follow if we can show that

$$
\sup _{B} \sum_{j: j(1-\theta)+L(B) \geq 0} \int_{|x| \gtrsim 2^{L(B)}}\left|\mathcal{K}_{m_{j}}(x)\right| d x<\infty .
$$

In fact,

$$
\begin{gathered}
\int_{|x| \geq 2^{L(B)}}\left|\mathcal{K}_{m_{j}}(x)\right| d x=\int_{|x| \geq 2^{j+L(B)}}\left|\mathcal{K}_{m^{j}}(x)\right| d x \\
2^{(Q / 2-s)(j+L(B))} \int_{G}\left|K_{m^{j}}(x)\right|^{2}\left(1+|x|^{s}\right)^{2} d x \lesssim 2^{-(s-Q / 2)(j(1-\theta)+L(B))}
\end{gathered}
$$


PAOLO CIATTI AND JAMES WRIGHT

and this sums in $j$ with $j(1-\theta)+L(B) \geq 0$ if $s>Q / 2$, uniformly in $B$. Here we used (18) and the $L^{2}$ Sobolev condition in (13) in the penultimate inequality. This establishes (25) and completes the proof of the weak-type $(1,1)$ bound in Theorem 3.3 .

\section{The proof of Theorem 3.3 - the Hardy space bound}

Elements in the Hardy space $H^{1}(G)$ have an atomic deomposition (see [10]) and so it suffices to fix an atom $a_{B}$ supported in a ball $B$ and prove

$$
\int_{G}\left|m(\sqrt{\mathcal{L}}) a_{B}(x)\right| d x \lesssim 1
$$

for our spectral multiplier $m \in \mathcal{M}_{Q}$.

Without loss of generality we may assume that the ball $B$ is centred at the origin. The $L^{2}$ boundedness of $m(\sqrt{\mathcal{L}})$ implies that $\int_{|x| \leq C 2^{L}}\left|m(\sqrt{\mathcal{L}}) a_{B}(x)\right| d x \lesssim 1$ via the Cauchy-Schwarz inequality and so it suffices to show that

$$
\int_{|x| \gg 2^{L}}\left|m(\sqrt{\mathcal{L}}) a_{B}(x)\right| d x \lesssim 1
$$

holds where $2^{L}$ is the radius of the ball $B$.

From our basic decomposition $m=m_{\text {small }}+m_{\text {large }}$, it suffices as before to treat the operator $T:=\sum_{j: j \theta>0} m_{j}(\sqrt{\mathcal{L}})$ and show that (27) holds with $m(\sqrt{\mathcal{L}})$ replaced by $T$.

We bound the integral in (27) by

$$
\sum_{j \in N} \int_{|x| \gg 2^{L}}\left|\mathcal{K}_{m_{j}} * a_{B}(x)\right| d x+\sum_{j \in P} \int_{|x| \gg 2^{L}}\left|\mathcal{K}_{m_{j}} * a_{B}(x)\right| d x=: I+I I
$$

where $N=\{j: j \theta>0, j(1-\theta)+L \leq 0\}$ and $P$ denotes the complementary range.

For $j \in P$, we note that when $|x| \gg 2^{L}$,

$$
\mathcal{K}_{m_{j}} * a_{B}(x)=\int_{|y| \leq 2^{L}} \mathcal{K}_{m_{j}}\left(x y^{-1}\right) a_{B}(y) d y=\int_{G} \mathcal{K}_{m_{j}}\left(x y^{-1}\right) \chi_{E_{L}}\left(x y^{-1}\right) a_{B}(y) d y
$$

where $E_{L}=\left\{x \in G:|x| \geq 2^{L}\right\}$. Hence if we denote by $K=\mathcal{K}_{m_{j}}(x) \chi_{E_{L}}(x)$,

$$
\begin{gathered}
\int_{|x| \gg 2^{L}}\left|\mathcal{K}_{m_{j}} * a_{B}(x)\right| d x=\int_{G}\left|K * a_{B}(x)\right| d x \leq \int_{G}|K(x)| d x=\int_{|x| \geq 2^{L}}\left|\mathcal{K}_{m_{j}}(x)\right| d x \\
=\int_{|x| \geq 2^{j+L}}\left|\mathcal{K}_{m^{j}}(x)\right| d x=\int_{|x| \geq 2^{j+L}}\left|\mathcal{K}_{m^{j}}(x)\right| \frac{1+|x|^{s}}{1+|x|^{s}} d x \\
\leq 2^{-(s-Q / 2)(j+L)} \sqrt{\int_{G}\left|\mathcal{K}_{m^{j}}(x)\right|^{2}\left(1+|x|^{s}\right)^{2} d x} \lesssim 2^{-(s-Q / 2)(j(1-\theta)+L)}
\end{gathered}
$$

where in the last inequality we used (18) with some $s>Q / 2$ and the $L^{2}$ Sobolev condition of our multiplier $m$ as stated in (13). Since $\theta \neq 1$, this shows that $I I$ is uniformly bounded 
For $I$, we split $N=N_{1} \cup N_{2}$ further such that $N_{1}=\{j \in N: j+Q \leq 0\}$ and $N_{2}=\{j \in N: j+Q>0\}$. This splits $I=I_{1}+I_{2}$ accordingly.

For the sum over $j \in N_{1}$, we will use the cancellation of the atom $a_{B}$ : for $j \in N_{1}$,

$$
\int_{|x| \gg 2^{L}}\left|\mathcal{K}_{m_{j}} * a_{B}(x)\right| d x \leq \int_{G}\left|a_{B}(y)\right|\left[\int_{C 2^{j+L} \leq|x|}\left|\mathcal{K}_{m^{j}}\left(x\left(\delta_{2^{j}} y\right)^{-1}\right)-\mathcal{K}_{m^{j}}(x)\right| d x\right] d y
$$

and so by applying the mean value theorem on stratified groups (see (16)), we see that the inner integral on the right hand side is at most

$$
2^{j+L} \int_{2^{j+L} \leq|x|} \sup _{1 \leq r \leq k}\left|X_{r} \mathcal{K}_{m^{j}}(x)\right| d x
$$

and so

$$
\int_{|x| \gg 2^{L}}\left|\mathcal{K}_{m_{j}} * a_{B}(x)\right| d x \leq 2^{j+L} \sum_{r=1}^{k} \int_{2^{j+L} \leq|x|}\left|X_{r} \mathcal{K}_{m^{j}}(x)\right| d x .
$$

Let $X$ denote one of the $X_{r}$ 's - our immediate goal is to show that the bound

$$
\int_{2^{j+L} \leq|x|}\left|X \mathcal{K}_{m^{j}}(x)\right| d x \leq C
$$

holds, uniformly for all $j$ and $L$. If this is the case, then we see that

$$
I_{1}=\sum_{j \in N_{1}} \int_{|x| \gg 2^{L}}\left|\mathcal{K}_{m_{j}} * a_{B}(x)\right| d x \lesssim \sum_{j \in N_{1}} 2^{j+L} \lesssim 1,
$$

completing the analysis for $I_{1}$.

To show (28), we will use (19) for two different values of $s$. We split the integral in (28) into two parts:

$$
\int_{2^{j+L} \leq|x| \leq 2^{j+L+\Lambda}}\left|X \mathcal{K}_{m^{j}}(x)\right| d x+\int_{2^{j+L+\Lambda} \leq|x|}\left|X \mathcal{K}_{m^{j}}(x)\right| d x=: S_{\Lambda}+L_{\Lambda}
$$

for some large $\Lambda>0$ to be chosen appropriately.

For $S_{\Lambda}$ we use (19) with some $s_{*}<Q / 2$ : by Cauchy-Schwarz,

$$
S_{\Lambda}^{2} \leq 2^{2\left(Q / 2-s_{*}\right)(j+L+\Lambda)} \int_{G}\left|X \mathcal{K}_{m^{j}}(x)\right|^{2}\left(1+|x|^{s_{*}}\right)^{2} d x
$$

and so using (19) and the $L^{2}$ Sobolev condition (13) of our multiplier $m$,

$$
S_{\Lambda} \lesssim 2^{\left(Q / 2-s_{*}\right)(j(1-\theta)+L)} 2^{\left(Q / 2-s_{*}\right) \Lambda}
$$

In a similar way, using (19) with some $s>Q / 2$, we have

$$
L_{\Lambda}^{2} \leq 2^{-2(s-Q / 2)(j+L+\Lambda)} \int_{G}\left|X \mathcal{K}_{m^{j}}(x)\right|^{2}\left(1+|x|^{s}\right)^{2} d x
$$

and so using the $L^{2}$ Sobolev condition (13) of our muliplier $m$, we see that

$$
L_{\Lambda} \lesssim 2^{-(s-Q / 2)(j(1-\theta)+L)} 2^{-(s-Q / 2) \Lambda} .
$$

Optimising the two estimates gives $\Lambda=-(j(1-\theta)+L)$ which is positive since $j \in N$. Hence with this choice of $\Lambda, S_{\Lambda}+L_{\Lambda} \lesssim 1$, establishing (28) and completing the analysis for $I_{1}$. 
Finally we turn to $I_{2}$ where $j \in N_{2}$ implies $j+L \geq 0$. Here it does not make sense to use the cancellation of the atom $a_{B}$. Instead we use our knowledge of the $L^{2}$ size of $a_{B} ;\left\|a_{B}\right\|_{L^{2}(G)} \leq|B|^{-1 / 2}=2^{-L Q / 2}$. We begin by splitting the integral into two parts as above:

$$
\int_{2^{L} \leq|x| \leq 2^{L+\Lambda}}\left|\mathcal{K}_{m_{j}} * a_{B}(x)\right| d x+\int_{|x| \geq 2^{L+\Lambda}}\left|\mathcal{K}_{m_{j}} * a_{B}(x)\right| d x:=S_{\Lambda}+L_{\Lambda}
$$

for some appropriate $\Lambda$. For $S_{\Lambda}$, we use the $L^{\infty}$ condition in (13) and CauchySchwarz to see that

$$
S_{\Lambda} \leq 2^{(L+\Lambda) Q / 2}\left\|\mathcal{K}_{m_{j}} * a_{B}\right\|_{L^{2}} \leq 2^{(L+\Lambda) Q / 2} 2^{-j \theta Q}\left\|\Phi_{j} * a_{B}\right\|_{L^{2}} \leq 2^{\Lambda Q / 2} 2^{-j \theta Q / 2} .
$$

On the other hand, for $L_{\Lambda}$, we have

$$
\begin{gathered}
L_{\Lambda} \leq \int_{|x| \geq 2^{L+\Lambda}}\left|\mathcal{K}_{m_{j}}(x)\right| d x=\int_{|x| \geq 2^{j+L+\Lambda}}\left|\mathcal{K}_{m^{j}}(x)\right| d x \\
\leq 2^{-(s-Q / 2)(j+L+\Lambda)} \sqrt{\int_{G}\left|\mathcal{K}_{m^{j}}(x)\right|^{2}\left(1+|x|^{s}\right)^{2} d x} \lesssim 2^{-(s-Q / 2)(j(1-\theta)+L+\Lambda)}
\end{gathered}
$$

by (19) with $s>Q / 2$ and Cauchy-Schwarz. Optimising the two estimates gives $\Lambda$ with $2^{s \Lambda}=2^{-(s-Q / 2)(j+L)} 2^{j \theta s / 2}$ which is positive since $j \in N$. Hence with this choice of $\Lambda, S_{\Lambda}+L_{\Lambda} \lesssim 2^{-Q / 2(1-Q / 2 s) /(j+L)}$ which is summable over $j \in N_{2}$ since $j+L>0$, showing that

$$
I_{2}=\sum_{j \in N_{2}} \int_{2^{L} \leq|x|}\left|\mathcal{K}_{m_{j}} * a_{B}(x)\right| d x
$$

is uniformly bounded in $L$ and this completes the analysis for $I_{2}$, establishing (27) and hence (26).

This finishes the $H^{1}(G)$ bound of $m(\sqrt{\mathcal{L}})$ and hence the proof of Theorem 3.3

\section{REFERENCES}

[1] G. Alexopoulos, Oscillating multipliers on Lie groups and Riemannian manifolds, Tôhoku Math. J. 46 (1994), 457-468.

[2] S. Chanillo, Weighted norm inequalities for strongly singular convolution operators, Trans. Amer. Math. Soc. 281, no.1 (1984), 77-107.

[3] S. Chanillo, D. Kurtz and G. Sampson, Weighted $L^{p}$ estimates for oscillating kernels, Ark. Mat. 21 (1983), 233-257.

[4] S. Chanillo, D. Kurtz and G. Sampson, Weighted weak $(1,1)$ and weighted $L^{p}$ estimates for oscillating kernels, Trans. Amer. Math. Soc. 295, no. 1 (1986), 127-145.

[5] M. Christ, $L^{p}$ bounds for spectral multipliers on nilpotent groups, Trans. Amer. Maths. Soc. 328, no. 1 (1991), 73-81.

[6] M. Christ, Weak type $(1,1)$ bounds for rough operators, Annals of Mathematics 128 (1988), 19-42.

[7] M. Cowling, S. Giulini and S. Meda, Oscillatory integrals related to the wave equation on noncompact symmetric spaces, J. London Math. Soc. 66 (2002), 691-702.

[8] C. Fefferman, Inequalities for strongly singular integral operators, Acta Math. 24 (1970), 9-36.

[9] C. Fefferman and E.M. Stein, $H^{p}$ spaces of several variables, Acta Math. 129 (1972), 137-193.

[10] G. Folland and E.M. Stein, Hardy spaces on homogeneous groups, Math. Notes 28, Princeton University Press (1982). 
[11] S. Giulini and S. Meda, Oscillating multipliers on noncompact symmetric spaces, Journal fur die reine und angewandte Mathematik 409 (1990), 93-105.

[12] L. Grafakos, L. Liu and D. Yang, Vector-valued singular integrals and maximal functions on spaces of homogeneous type, Math. Scand. 104 (2009), 296-310.

[13] W. Hebisch, Multiplier theorem on generalized Heisenberg groups, Colloquium Mat. 65, no. 2 (1993), 231-239.

[14] I.I. Hirschman, On multiplier transformations, Duke Math. J. 26 (1959), 222-242.

[15] L. Hörmander, Pseudo-differential operators and hypoelliptic equations, Proc. Sympos. Pure Math., 10, Amer. Math. Soc. (1967), 138-183.

[16] A. Hulanicki, A functional calculus for Rockland operators on nilpotent Lie groups, Studia Math. 78 (1984), no. 3, 253-266.

[17] A. Martini, Joint functional calculi and a sharp muliplier theorem for the Kohn Laplacian on the sphere, Math. Z. 286 (2017), 15391574.

[18] A. Martini and Detlef Müller, Spectral multipliers of euclidean type on new classes of two-step stratified groups, Proc. London Math. Soc. 109 (2014), 1229-1263.

[19] A. Martini and Detlef Müller, Spectral multipliers on 2-step groups: topological versus homogeneous dimension, GAFA 26 (2016), 680-702.

[20] G. Mauceri and S. Meda, Vector-valued multipliers on stratified groups, Revista Ibero. Mat. 6, no 3 (1990), 141-154.

[21] A. Miyachi, On the weakly strongly singular integrals, Japan J. Math. 4 (1978), 221-262.

[22] A. Miyachi, On some estimates for the wave equation in $L^{p}$ and $H^{p}$, J. Fac. Sci. Tokyo Sci. IA 27 (1980), 331-354.

[23] D. Müller and A. Seeger, Sharp $L^{p}$-bounds for the wave equation on groups of Heisenberg type, Analysis and PDE, 8 (2015), no. 5, 1051-1100.

[24] D. Müller and E.M. Stein, On spectral multipliers for Heisenberg and related groups, J. Math. Pures Appl 73 (1994), 413-440.

[25] D. Müller and E.M. Stein, $L^{p}$-estimates for the wave equation on the Heisenberg group, Revista Mat. Ibero. 15 (1990), 297-334.

[26] J. Peral, $L^{p}$ estimates for the wave equation, J. Functional Analysis 36 (1980), 114-145.

[27] A. Seeger, C. Sogge and E.M. Stein, Regularity properties of Fourier integral operators, Annals of Math. 103 (1991), 231-251.

[28] A. Sikora, Multiplier theorem for sub-Laplacians on homogeneous groups, C. R. Acad. Sci. Paris Sr. I Math. 315 (1992), no. 4, 417-419.

[29] E.M. Stein, Harmonic Analysis, Princeton University Press (1993).

[30] E.M Stein, Singular integrals, harmonic functions and differentiability properties of functions of several variables, Proc. Syrmp. in Pure Math., 10 (1967), 316-335.

[31] S. Wainger, Special trigonometric series in $k$ dimesnions, Mem. Amer. Math. Soc., no. 59 (1965).

[32] A. Zygmund, Trigonometric series (2 vols), 2nd ed., Cambridge Univ. Press, London and New York (1977).

Università degli Studi di Padova, Via Marzolo 9, 35131 Padova, Italia

E-mail address: paolo.ciatti@unipd.it

Maxwell Institute of Mathematical Sciences and the School of Mathematics, UniverSity of Edinburgh, JCMB, The King's Buildings, Peter Guthrie Tait Road, Edinburgh, EH9 3FD, SCOTLAND

E-mail address: J.R.Wright@ed.ac.uk 